\title{
Study of Leukopenia as haematological parameter, to find it's diagnostic rationale in dengue patients
}

\author{
Manoj Kumar Gupta ${ }^{1}$, Jagjeevan Ram ${ }^{2, *}$, Poonam Rani ${ }^{3}$, Kamlesh Kumar ${ }^{4}$, Amrita \\ ${ }^{\mathbf{1}}$ Assistant Professor, ${ }^{\mathbf{2}}$ Associate Professor, ${ }^{\mathbf{3}, 4,5}$ Lecturer, Dept. of Pathology, ${ }^{\mathbf{1 , 3}, \mathbf{4}, \mathbf{5}}$ M.R.A. Medical College, Ambedkar Nagar, \\ Uttar Pradesh, ${ }^{2}$ Ganesh Shankar Vidyarthi Memorial Medical College, Kanpur, Uttar Pradesh, India
}

*Corresponding Author: Jagjeevan Ram

Email: dr.jrt4@gmail.com

Received: $20^{\text {th }}$ February, 2018

Accepted: $24^{\text {th }}$ April, 2018

\begin{abstract}
Introduction: The dengue fever is in the form of epidemic in India which occurs usually after rainy season. There is need of adjuvant haematological parameters that can at very initial stage differentiate the dengue fever from other viral fever which are prevalent at the same time.

Aims and Objectives: This retrospective study was carried out to find association of leukopenia with dengue fever. As screening test for dengue fever are not available in remote areas, so we had tried to access diagnostic power of leukopenia in dengue fever along with thrombocytopenia.

Materials and Methods: Total 520 cases had been taken. Out of these 220 cases were positive for dengue fever as confirmed on dengue fever ELISA test for NS1 and/or IgM. Retrieved data were analysed for platelet counts and total leukocyte counts.

Results: In Total 220 cases of dengue fever, 101 cases had shown both leukopenia and thrombocytopenia. While in 300 normal cases there were only 2 cases of leukopenia along with thrombocytopenia. The sensitivity of leukopenia alone for diagnosis of dengue fever was only $50.91 \%$, but the specificity was $98.33 \%$. Positive Likelihood Ratio (PLR) of leukopenia to dengue fever was 30.55 . Although the sensitivity was decreased to $45.91 \%$ if leukopenia with thrombocytopenia was used as diagnostic tool for dengue fever, but specificity was increased up to $99.33 \%$ with PLR up to 68.86 . The total leukocyte count and platelets were statistically reduced with extreme significance $(P<0.001)$ in dengue fever patients as compared to normal age and gender matched healthy control. The correlation coefficient was +0.34 which was considered as significant at the level of $p<0.01$. Conclusion: The leukopenia alone is highly specific for dengue fever, but if combined with thrombocytopenia, specificity is further increased. Both Leukopenia and thrombocytopenia were statistically extremely significant $(P<0.001)$ in dengue fever. There is high correlation between severities of thrombocytopenia with the degree of leukopenia.
\end{abstract}

Keywords: Dengue fever, Leukopenia, ELISA, Platelet count, Thrombocytopenia.

\section{Introduction}

In India dengue fever is prevalent in India especially in north region. It is more prevalent from September to December, after the rainy season. In 2017 total $1,57,220$ cases have been reported in India with 3032 cases in UP according to data available on web site of National Vector Borne Disease Control Programme, India. ${ }^{9}$ These positive data are based on dengue fever ELISA tests, which are largely institutional based. ${ }^{10}$ But the diagnosis of dengue fever is largely based on dengue fever rapid card tests in non institutional hospitals or clinics. So there is need of additional supporting parameter besides thrombocytopenia that confirms or correlates with the reactive results, based on rapid cards. In our study we had emphasized the role of "leukopenia" as additional haematological parameter which could be used as adjuvant to thrombocytopenia. A retrospective, hospital-based study, which was conducted at a university hospital in southern, enlightened with its conclusion that leukopenia along with thrombocytopenia could be used as useful predictive markers for early diagnosis of dengue fever infection. ${ }^{7}$ In a study by Lin et al showed that there was significant leukopenia in $38 \%$ of cases of confirmed cases of dengue fever. After fever onset the leukocytes reached nadir of $1000-2000 / \mathrm{mm}^{3}$ at $5^{\text {th }}$ to $6^{\text {th }}$ day and thrombocytes reached nadir $20000-50000 / \mathrm{mm}^{3}$ at $5^{\text {th }}$ to $7^{\text {th }}$ day. This study suggested that leukopenia in dengue fever might be caused by virus-induced destruction or inhibition of myeloid progenitor cells. ${ }^{1}$ One study by James A et al found that patients with dengue fever patients had significantly reduced platelets count and lower leukocytes counts along with neutrophil counts, and a higher frequency of petechiae than other patients of febrile illness. ${ }^{11}$ Francisca Raimunda F et al studied the laboratory tests, both non-specific [blood count, platelet count, tourniquet test, prothrombin time (PT), activated partial thromboplastin time (APTT), liver function tests and serum albumin concentration] and specific tests (viral isolation tests and serology for antibody examination). Most prominent hematological change, they had observed was leukopenia as well as sometimes mild leukocytosis at the onset of the disease, with neutrophilia. ${ }^{12}$

\section{Materials and Methods}

This study was carried out in total 520 patient with chief complain of high grade fever since last 5 days with significant arthalgia. The presence of rash was not 
included in our study because this sign would have decreased the number of dengue fever negative cases. All the 520 patients were subjected for dengue fever NS1 ELISA as well as IgM ELISA. Patient were considered positive with any of or both NS1 ELISA positivity and IgM ELISA positivity. We had found total 220 cases as dengue fever positive. The haematological data i.e. complete blood count was analysed for each patient. Out of different haematological parameters we had considered leukopenia and thrombocytopenia in our study. The leukopenia was defined as total leukocyte count below 4000 cells/cumm and thrombocytopenia was considered when platelet counts were less than $1.5 \mathrm{lac} / \mathrm{cumm} .{ }^{8}$ The data was analysed by using SPSS 23.0 for windows software.

\section{Result and Discussion}

The total leukocyte count (TLC) and platelets were statistically extremely significantly decreased $(P$ $<0.001)$ in dengue fever patients as compared to normal age and gender matched healthy control. [Table 5]

Correlation of Leukopenia with Thrombocytopenia: We had also tried the find out whether there was any correlation between the degrees of thrombocytopenia with the degree of leukopenia. The correlation analysis between degrees of thrombocytopenia with severity of leukopenia was carried out in 112 positive cases. The correlation coefficient was +0.34 . Which was considered as significant at the level of $p<0.01$. So it was concluded that there was high correlation between severities of thrombocytopenia with the degree of leukopenia.

Studies on severe neutropenia and Leukopenia in dengue fever were scarce, and its clinical significance is still uncertain. 1,921 RTPCR confirmed adult dengue fever patients were admitted to the CDC, Singapore between 2005 and 2008. It had been found that severe neutropenia, defined as absolute neutrophilic count (ANC) $\leq 5000 /$ cumm, was found in $11.8 \%$ on first day. ANC nadir occurred on illness day 5. But severe neutropenia was not predictive of more severe disease and not associated with secondary bacterial infections, prolonged hospital stay, prolonged fever, or fatal outcome. ${ }^{3} 56(26.6 \%)$ patients out of 216 dengue fever IgM positive cases showed leukopenia and $161(77.1 \%)$ patients showed neutropenia in retrospective observational study done at AKU university in Karanchi. $^{2}$ Majority of patients were discharged without any adverse reaction. It was concluded that haematological parameter were also important clue and should be tested, when a patient came with sign and symptoms of dengue fever.

The role of immunological factors in the pathogenesis of Dengue fever Hemorrhagic Fever (DHF), particularly Dengue fever Shock Syndrome (DSS) was also studied. The complement activity, circulating immune complex (IC), histamine level, platelets and leucocytes were determined in the blood of 30 patients and 43 healthy persons. Besides other parameters, the leukocyte count was significantly reduced $(\mathrm{P}<0.001)$. Furthermore, a marked correlation was found between the changes of the above parameters and the clinical stages of the disease. ${ }^{4}$ In the DENV-1 study among the clinically apparent dengue fever patients the mean leukocyte count was 3000/cumm \pm SD 780/cumm. Although minimum leukocyte counts $(P=0.28)$ and the duration of leukopenia $(P=0.55) \mathrm{did}$ not reveal significant correlation. ${ }^{5}$

Leukopenia was frequently found and may be accompanied by varying degrees of thrombocytopenia. ${ }^{6}$ Tzong-Shiann et al suggested that leukopenia along with thrombocytopenia was useful for early diagnosis of dengue fever infection. The most notable laboratory findings in confirmed dengue fever case were included leukopenia $(2966 \pm 1896 /$ cumm), thrombocytopenia $\left(102 \pm 45 \times 10^{3} / \mathrm{cumm}\right)$. The positive predictive value (PPV) was high for combination of leukopenia with thrombocytopenia. ${ }^{7}$ In the study conducted by Francisca Raimunda $\mathrm{F}$ et al leukopenia was the most prominent hematological change, sometimes with counts of less than 2000/cumm, as shown in our study also in which there were $51 \%$ cases were of leukopenia with 15 patients having leukocytes less than 2000/cumm. ${ }^{12}$

In present study leukopenia as single parameter had shown high specificity $(98.33 \%)$ and low PLR. [Table 2] The specificity along with PLR was more increased if the leukopenia was combined with thrombocytopenia. [Table 4] The t-test showed that the reduction in both total leukocyte count and platelets were statistically extremely significantly $(P<0.001)$ in dengue fever patients as compared to normal age and gender matched healthy control. [Table 5] The correlation analysis of degree of thrombocytopenia with severity of leukopenia showed the correlation coefficient as +0.34 , which is considered as significant at the level of $p<0.01$ suggesting a high correlation between them.

Table 1: Details of total 520 cases with leukocyte count and platelets counts

\begin{tabular}{|l|c|c|c|c|c|}
\hline & $\begin{array}{c}\text { Number \& } \\
\text { \% }\end{array}$ & $\begin{array}{c}\text { Leukocyte count } \\
\text { per cumm }\end{array}$ & $\begin{array}{c}\text { Number \& } \\
\text { \% }\end{array}$ & $\begin{array}{c}\text { Platelets count } \\
\text { per cumm }\end{array}$ & $\begin{array}{c}\text { Number \& } \\
\text { \% }\end{array}$ \\
\hline Dengue fever positive & 220 & $<4000$ & $112(51 \%)$ & $<1.5$ lac & 196 \\
& $(43 \%)$ & $>4000$ & $108(49 \%)$ & $>1.5$ lac & $\begin{array}{c}24 \\
(89 \%)\end{array}$ \\
\cline { 3 - 6 } & & & & & \\
& & & & &
\end{tabular}

Indian Journal of Pathology and Oncology, October-December, 2018;5(4):609-612 


\begin{tabular}{|l|c|c|c|c|c|}
\hline $\begin{array}{l}\text { Dengue fever negative } \\
\text { (Control) }\end{array}$ & $\begin{array}{c}300 \\
(57 \%)\end{array}$ & $<4000$ & $05(1.6 \%)$ & $<1.5$ lac & $\begin{array}{c}22 \\
(7.3 \%)\end{array}$ \\
\cline { 3 - 5 } & & $>4000$ & $\begin{array}{c}195 \\
(98.4 \%)\end{array}$ & $>1.5$ lac & $\begin{array}{c}278 \\
(92.7)\end{array}$ \\
\hline
\end{tabular}

Table 2: Evaluation of leukopenia alone as diagnostic test

\begin{tabular}{|l|c|}
\hline \multicolumn{1}{|c|}{ Statistic } & Value \\
\hline Sensitivity & $50.91 \%$ \\
\hline Specificity & $98.33 \%$ \\
\hline Positive Likelihood Ratio & 30.55 \\
\hline Negative Likelihood & 0.50 \\
\hline
\end{tabular}

Table 3: Combined parameters study

\begin{tabular}{|l|c|c|}
\hline Platelets + TLC & Dengue fever positive cases & - Dengue fever negative cases \\
\hline$<1.5+<4000$ & 101 & 02 \\
\hline$<1.5+>4000$ & 95 & 20 \\
\hline$>1.5+<4000$ & 11 & 03 \\
\hline$>1.5+>4000$ & 13 & 275 \\
\hline Total & 220 & 300 \\
\hline
\end{tabular}

Table 4: Evaluation of leukopenia combined with thrombocytopenia as diagnostic test

\begin{tabular}{|l|c|}
\hline \multicolumn{1}{|c|}{ Statistic } & Value \\
\hline Sensitivity & $45.91 \%$ \\
\hline Specificity & $99.33 \%$ \\
\hline Positive Likelihood Ratio & 68.86 \\
\hline Negative Likelihood & 0.54 \\
\hline
\end{tabular}

Table 5: Comparative study of total leukocyte count in dengue fever patients

\begin{tabular}{|l|c|c|c|}
\hline & $\begin{array}{c}\text { Patient } \\
\text { (Mean +/- SD }\end{array}$ & $\begin{array}{c}\text { Control } \\
\text { Mean +/- SD }\end{array}$ & \multirow{2}{*}{$<0.001$} \\
\hline No of cases & 220 & 300 & \multirow{2}{*}{ TLC (cells/cumm) } \\
\cline { 1 - 3 } Platelet & $5383+/-3116$ & $8308.0+/-2639$ & \\
\hline
\end{tabular}

\section{Conclusion}

There is significant reduction in leukocyte count as well as platelets count in dengue fever patients and high correlation between severities of thrombocytopenia with the degree of leukopenia. We have concluded our study that the duo of leukopenia and thrombocytopenia can be used as good negative predictor for dengue fever.

\section{References}

1. Lin SF, Liu HW, Chang CS, Yen JH, Chen TP. "Hematological aspects of dengue fever". Gaoxiong $Y i$ Хие Кe Хие Zhi. 1989;5(1):12-6.

2. Ali N, Usman M, Syed N, Khurshid M. "Haemorrhagic manifestations and utility of haematological parameters in dengue fever: a tertiary care centre experience at Karanchi”. Scand J Infect Dis. 2007;39(11-12):1025-8.

3. Tun-Linn Thein David C. Lye, Yee-Sin Leo, Joshua G. $\mathrm{X}$. Wong, Ying Hao, and Annelies Wilder-Smith. "Severe Neutropenia in Dengue fever Patients: Prevalence and Significance". Am J Trop Med Hyg. 2014;90(6):984-987.

4. Phan DT, Ha NT, Thuc LT, Diet NH, Phu LV, Ninh LY, An VT. "Some changes in immunity and blood in relation to clinical states of dengue fever hemorrhagic fever patients in Vietnam". Haematologia (Budap). 1991;24(1):13-21.

5. Hiroshi Nishiura Scott B. Halstead "Natural History of Dengue fever Virus (DENV) -1 and DENV -4

Infections: Reanalysis of Classic Studies". The Journal of Infectious Diseases. 2007;195(7):1007-10.

6. Centers for disease control and prevention. Office of Us department of health and human services. "Clinical guidance-Dengue fever". Available from https://www.cdc.gov/dengue fever/clinicallab/clinical.html. (Accessed 14 ${ }^{\text {th }}$ Feb 2018).

7. Tzong-Shiann Ho, Shih-Min Wang, Yee-Shin Lin and Ching-Chuan Liu. "Clinical and laboratory predictive markers for acute dengue fever infection". Journal of Biomedical Science. 2013;20:75.

8. Kumar V, et al. "Diseases of white blood cells, lymph nodes, spleen, and thymus". Robbins and Cotran Pathologic Basis of Disease. 9th ed. Philadelphia, Pa.: Saunders Elsevier; 2015. http://www.clinicalkey.com. Accessed Nov. 18, 2015.

9. National Vector Borne Disease Control Programme. Directorate General of Health Services Ministry of Health $\&$ Family Welfare. Dengue Cases and Deaths in the Country since 2010. http://www.nvbdcp.gov.in/dencd.html (Accessed 17 $7^{\text {th }}$ Feb 2018).

10. Geneva: World Health Organization; 2009. Dengue: Guidelines for Diagnosis, Treatment, Prevention and 
Control: New Edition. Laboratory diagnosis and diagnostic tests.

https://www.ncbi.nlm.nih.gov/books/NBK143156. (Accessed 20 $0^{\text {th }} \mathrm{Feb} 2018$ ).

11. James A. Potts and Alan L. Rothman. "Clinical and laboratory features that distinguish dengue from other febrile illnesses in endemic populations". Trop Med Int Health. 2008;13(11):1328-1340.

12. Francisca Raimunda F. Guerreiro Azin. Dengue: profile of hematological and biochemical dynamics. Rev Bras Hematol Hemoter. 2012;34(1):36-41.

How to cite this article: Gupta M. K, Ram J, Rani P, Kumar K, Amrita. Study of Leukopenia as haematological parameter, to find it's diagnostic rationale in dengue patients. Indian $\mathrm{J}$ Pathol Oncol. 2018;5(4):609-612. 